\title{
$1 \quad$ Candida auris is rendered non-viable by medium-chain fatty acids
}

2 Running title: Susceptibility of $C$. auris to medium-chain fatty acids

4 Kalynne R. Green, ${ }^{\mathrm{a}}{ }^{\dagger}$ Magdia De Jesus, ${ }^{\mathrm{b}, \mathrm{c}} \S$ and Kearney T. W. Gunsalus ${ }^{\mathrm{a}} \#$

$5 \quad$ aDepartment of Biology, Siena College, Loudonville, NY

$6{ }^{b}$ Department of Biomedical Sciences, University at Albany School of Public Health, Albany, $7 \quad N Y$

8 'Division of Infectious Diseases, Wadsworth Center, New York State Department of Health, $9 \quad$ Albany, $N Y$

10 †Present address: Marist College Physician Assistant Program, Poughkeepsie, NY

11 §Present address: Bacterial Vaccines Research and Technology, Pfizer Inc., Pearl River, $12 \quad N Y$

13 *Present address: Department of Basic Sciences, Augusta University/University of Georgia 14 Medical Partnership, Athens, GA

15 \# Address correspondence to Kearney T. W. Gunsalus, gunsalus@uga.edu

\section{Abstract}

18 The medium-chain fatty acids, octanoic and decanoic acid, found in coconut oil, were

19 fungistatic and decanoic acid was fungicidal against a panel of Candida auris strains, during 20 both planktonic and biofilm growth. The strains were from all four major geographic clades, 21 and some were resistant to several classes of antifungal drugs. These compounds are safe, 22 natural products and could provide a new strategy for skin decolonization and environmental 23 decontamination. 
Keywords Candida, Candida auris, medium-chain fatty acid, antifungal agents, antifungal susceptibility testing, pathogenic yeasts, emerging pathogen, biofilm

Candida auris has rapidly emerged around the world as a drug-resistant fungal pathogen causing hospital outbreaks with high mortality rates. Factors contributing to its virulence likely include $C$. auris' persistence both as a skin colonizer and in the environment $(1,2)$; its ability to transfer to other patients or healthcare workers; and its resistance to various disinfection measures and antifungal drugs [reviewed in $(3,4)]$.

While the drug susceptibility profile varies between strains, and particularly between clades, many strains of $C$. auris are resistant to at least two of the three major classes of antifungal drugs, and the emergence of pan-resistant strains occurs during treatment with antifungals [reviewed in $(3,5)]$.

There is an urgent need for new methods to achieve environmental decontamination and particularly skin decolonization, without using antifungal drugs. Coconut oil, its derivative medium-chain triglyceride (MCT) oil, and their constituent medium-chain fatty acids (MCFAs), have antimicrobial properties against other Candida species in vitro (6-9). We showed that dietary coconut oil reduces gastrointestinal colonization by Candida albicans in mice (10), and that dietary supplementation with MCT oil reduces Candida GI colonization in humans (11). Coconut oil is safe for human consumption and topical application; it is widely used in cooking and as a skin and hair care product. In this study, we investigated the antifungal activities of the medium-chain fatty acids, octanoic and decanoic acid, against Candida auris.

C. auris strains are susceptible to growth inhibition by medium-chain fatty acids. The antifungal activity of octanoic and decanoic acid was analyzed against a panel of strains of C. auris obtained from the Centers for Disease Control, specifically to be used in the laboratory of M. De Jesus (Table 1). The panel includes clinical isolates from four 

non-aggregate forming strains, and strains with resistance to azoles and amphotericin $\mathrm{B}$.

To work with the strains of $C$. auris, precautions were taken as previously described (2). The susceptibility of the $C$. auris strains to octanoic and decanoic acids was determined using a broth microdilution assay [(12) and supplemental methods]. Briefly, strains were seeded into 96-well plates in RPMI 1640 2\% glucose in the presence of a two-fold dilution series of octanoic or decanoic acid (final concentration $0.02-10 \mathrm{mM}$ ) and grown for 24 hours at $37^{\circ} \mathrm{C}$. Sealed plates were read with a microplate reader $(530 \mathrm{~nm})$, and results were expressed as a percentage of growth compared to the untreated cells.

Regardless of geographic clade or antifungal susceptibility profile, growth of all strains was inhibited by MCFAs. During planktonic growth, octanoic acid elicited 80\% growth inhibition (pMIC80) at 2.5-5 mM, and pMIC50 was $0.6-2.5 \mathrm{mM}$, depending upon the strain (Figure 1A). Decanoic acid is more potent, with a pMIC80 of $1.25-2.5 \mathrm{mM}$, and a pMIC50 of $0.6-1.25$ $\mathrm{mM}$ (Fig 1B).

Antifungal-resistant $\boldsymbol{C}$. auris strains are rendered non-viable by decanoic acid. To determine whether octanoic and decanoic acids are fungicidal, or merely fungistatic, cells from the wells treated with concentrations of octanoic and decanoic acids at or above the pMIC50 were serially diluted tenfold and spotted onto Sabouraud dextrose agar to determine whether viable cells could be recovered. While none of the tested concentrations of octanoic acid resulted in complete loss of viability, recovered colonies displayed variable colony morphology (particularly decreased colony size in some strains, Fig 2A). In contrast, decanoic acid was fungicidal, with minimum fungicidal concentrations (the lowest concentration showing no growth) of 5 or $10 \mathrm{mM}$ for all strains tested (Fig 2B-C).

Octanoic and decanoic acids reduce the viability of $C$. auris biofilms. Candida spp. biofilms tend to be highly resistant to antifungal drugs, even for strains that are drugsusceptible during planktonic growth. To determine the susceptibility of mature $C$. auris biofilms to octanoic and decanoic acids, $C$. auris biofilms were grown for 24 hours and then 
treated with octanoic or decanoic acid for an additional 24 hours. Cell viability was measured by metabolic activity of the cells using an XTT reduction assay (adapted from (13); supplemental methods). The sessile MIC50 (sMIC50) for octanoic acid was 2.5-20 mM for eight of the nine strains tested. All strains showed a sMIC50 of $1.25-5 \mathrm{mM}$, and sMIC80 of 5$10 \mathrm{mM}$, for decanoic acid (Fig 3).

Medium-chain fatty acids exhibited antifungal activity against $C$. auris isolates from all four major geographic clades; strains resistant to various classes of antifungal drugs, and both aggregate-forming and nonaggregating isolates. Non-aggregate-forming isolates have greater pathogenicity (14). Regardless of clade or antifungal resistance, all strains were susceptible to growth inhibition and were rendered non-viable by medium-chain fatty acids; moreover, the medium-chain fatty acids can greatly reduce the viability of $C$. auris biofilms. Biofilm formation tends to confer additional resistance to antifungals $(15,16)$, including via the upregulation of drug efflux pumps (17) and the sequestration of antifungals in the extracellular matrix (18). This broad susceptibility to MCFAs suggests that resistance to currently-available antifungal drugs does not confer resistance to MCFAs, and that the mechanisms of antifungal activity are different.

There is an urgent need for safe ways to reduce skin colonization by $C$. auris without using antifungals. This research suggests a new strategy to address this rising threat. MCFAs have been shown to have antimicrobial properties against a range of microbes, including other Candida species. The MCFAs found in coconut oil are safe for human consumption and topical application, even young and ill individuals. MCT oil, refined from coconut oil, is rich in MCFAs including octanoic and decanoic acid, is commercially available as a nutritional food, and already has multiple medical applications. The results of this study demonstrate that derivatives of coconut oil that are widely available and known to be safe, can greatly reduce the viability of a broad spectrum of $C$. auris strains, including strains from all four major geographic clades, and during both planktonic and biofilm growth. The efficacy of the MCFAs against a diverse panel of $C$. auris isolates argues for the exploration of 
MCFAs as a more general antifungal strategy — one that may prove particularly valuable given the global emergence of drug-resistant fungi such as $C$. auris.

\section{Materials and Methods}

Planktonic susceptibility assay

110 Strains were pre-cultured onto Sabouraud dextrose agar. Octanoic and decanoic acids were 111 obtained from Sigma Aldrich (O3907 and C1875) and stock solutions (2 M in 100\% ethanol)

112 were stored in glass vials at $-80^{\circ} \mathrm{C}$. A two-fold dilution series (2-0.004 M; final

113 concentrations $10-0.02 \mathrm{mM}$ ) of fatty acid was prepared in $100 \%$ ethanol, then diluted $1000 \mathrm{x}$

114 into 2x RPMI 1640 2\% G (2\% glucose; with L-glutamine and phenol red, without sodium bicarbonate; buffered to $\mathrm{pH} 7.0$ with 165 mM MOPS [3-(N-morpholino) propanesulfonic acid]), which was pre-warmed to increase solubility of fatty acids. $100 \mu \mathrm{L}$ was dispensed into each well of a flat-bottomed 96-well plate (CellTreat 229596); untreated wells contained an equivalent volume of ethanol (vehicle control). C. auris inocula were prepared by suspending a colony in sterile water and adjusting the $\mathrm{OD}_{530}$ to $0.011\left(\sim 1-5 \times 10^{5} \mathrm{CFU} / \mathrm{mL}\right) ; 100 \mu \mathrm{L}$ of inoculum was added to each well except the blanks (final inoculum $\sim 0.5-2.5 \times 10^{5} \mathrm{CFU} / \mathrm{mL}$ ). Plates were incubated in ambient air at $37^{\circ} \mathrm{C}$ for $24+/-2 \mathrm{~h}$, and $\mathrm{OD}_{530}$ was measured using a microplate reader (VersaMax, Molecular Devices). Data analysis was performed using $\mathrm{R}$ (20) and the tidyverse package (21).

Biofilm susceptibility assay

Liquid cultures in Sabouraud dextrose broth were grown overnight at $37^{\circ} \mathrm{C}, 150 \mathrm{rpm}$. Cells were pelleted via centrifugation, washed twice with and then resuspended in phosphate buffered saline (PBS, pH 7.4). The $\mathrm{OD}_{530}$ was adjusted to $0.11\left(\sim 1-5 \times 10^{6} \mathrm{CFU} / \mathrm{mL}\right)$ in RPMI $16402 \% \mathrm{G}$, and $100 \mu \mathrm{L}$ of inoculum was added to each well of a flat-bottom tissue culturetreated 96-well plate (Corning 3596), and incubated in ambient air at $37^{\circ} \mathrm{C}$ for 24 hours.

130 Medium was removed and wells washed with PBS to remove non-adherent cells. Biofilms were treated with a two-fold dilution series of octanoic or decanoic acid in RPMI $16402 \%$ G 
for 24 hours at $37^{\circ} \mathrm{C}$. Biofilms were washed with PBS; XTT solution $(0.5 \mathrm{~g} / \mathrm{L}, 1 \mu \mathrm{M}$ menadione, in PBS) was added, plates were incubated for $2-3$ hours at $37^{\circ} \mathrm{C}$ in the dark,

134 and absorbance at $490 \mathrm{~nm}$ was measured using a VersaMax microplate reader. The results

135 for each strain were expressed as a percentage of the $0 \mathrm{mM}$ fatty acid control. Data analysis was performed using $\mathrm{R}$ and tidyverse.

Figure Legends

Figure 1. C. auris strains are susceptible to growth inhibition by octanoic and octanoic or B) decanoic acid for 24 hours.

Figure 2. Minimum fungicidal concentration of decanoic and octanoic acids. Strains grown in the presence of A) $10 \mathrm{mM}$ octanoic acid, B) $10 \mathrm{mM}$ decanoic acid and C) $5 \mathrm{mM}$ decanoic acid were serially diluted ten-fold, spotted onto Sabaraud dextrose agar, and incubated at $37^{\circ} \mathrm{C}$ for 48 hours. (No additional colonies were observed after one week at 37 $\left.{ }^{\circ} \mathrm{C}.\right)$

Figure 3. Susceptibility of $C$. auris biofilms to octanoic and decanoic acids. Biofilms were grown for 24 hours, exposed to indicated concentrations of $\mathbf{A}$ ) octanoic or $\mathbf{B}$ ) decanoic acids for an additional 24 hours, and cell viability measured via XTT reduction.

\section{Acknowledgments}

154 We thank members of the De Jesus lab Steven Torres and Adina Longyear for their

155 technical assistance, and Dr. Navjot Singh of the Wadsworth Center Applied Genomic

156 Technologies Core for the helpful discussions of our data. We also thank Richard Cole of the 
157 Wadsworth Center Advanced Light Microscopy and Image Analysis Core. M. De Jesus

158 laboratory was supported by the University at Albany and Wadsworth Center start-up funds.

159 KRG was supported by the Summer Scholars program at Siena College. KTWG was

160 supported by a Summer Faculty Research Fellowship from the Siena College Committee on

161 Teaching and Faculty Development. The funders had no role in study design, data collection

162 and interpretation, or the decision to submit the work for publication. 
164 1. Piedrahita CT, Cadnum JL, Jencson AL, Shaikh AA, Ghannoum MA, Donskey CJ. 165 2017. Environmental surfaces in healthcare facilities are a potential source for transmission of Candida auris and other Candida species. Infect Control Hosp Epidemiol 38:1107-1109.

2. Torres SR, Kim HC, Leach L, Chaturvedi S, Bennett CJ, Hill DJ, De Jesus M. 2019. Assessment of environmental and occupational exposure while working with multidrug resistant (MDR) fungus Candida auris in an animal facility. J Occup Environ Hyg $16: 507-518$.

3. Bidaud AL, Chowdhary A, Dannaoui E. 2018. Candida auris: An emerging drug resistant yeast - A mini-review. J Mycol Medicale 28:568-573.

4. Forsberg K, Woodworth K, Walters M, Berkow EL, Jackson B, Chiller T, Vallabhaneni S. 2019. Candida auris: The recent emergence of a multidrug-resistant fungal pathogen. Med Mycol 57:1-12.

5. Lockhart SR. 2019. Candida auris and multidrug resistance: Defining the new normal. Fungal Genet Biol 131:103243.

6. Kabara JJ, Swieczkowski DM, Conley AJ, Truant JP. 1972. Fatty acids and derivatives as antimicrobial agents. Antimicrob Agents Chemother 2:23-28.

7. Bergsson G, Arnfinnsson J, Steingrímsson Ó, Thormar H. 2001. In vitro killing of Candida albicans by fatty acids and monoglycerides. Antimicrob Agents Chemother 45:3209-12.

8. Ogbolu DO, Oni AA, Daini OA, Oloko AP. 2007. In vitro antimicrobial properties of coconut oil on Candida species in Ibadan, Nigeria. J Med Food 10:384-387. 
9. Jadhav A, Mortale S, Halbandge S, Jangid P, Patil R, Gade W, Kharat K, Karuppayil SM. 2017. The dietary food components capric acid and caprylic acid inhibit virulence factors in Candida albicans through multitargeting. J Med Food 20:1083-1090.

10. Gunsalus KTW, Tornberg-Belanger SN, Matthan NR, Lichtenstein AH, Kumamoto CA. 2016. Manipulation of host diet to reduce gastrointestinal colonization by the opportunistic pathogen Candida albicans. mSphere 1:e00020-15.

11. Arsenault AB, Gunsalus KTW, Laforce-Nesbitt SS, Przystac L, DeAngelis EJ, Hurley ME, Vorel ES, Tucker R, Matthan NR, Lichtenstein AH, Kumamoto CA, Bliss JM. 2019. Dietary supplementation with medium-chain triglycerides reduces Candida gastrointestinal colonization in preterm infants. Pediatr Infect Dis J 38:164-168.

12. Arendrup MC, Meletiadis J, Mouton JW, Lagrou K, Hamal P, Guinea J, the Subcommittee on Antifungal Susceptibility Testing (AFST) of the ESCMID European Committee for Antimicrobial Susceptibility Testing (EUCAST). 2017. EUCAST definitive document E.DEF 7.3.1. Method for the determination of broth dilution minimum inhibitory concentrations of antifungal agents for yeasts. European Committee on Antimicrobial Susceptibility Testing.

13. Pierce CG, Uppuluri P, Tristan AR, Wormley FL, Mowat E, Ramage G, Lopez-Ribot JL. 2008. A simple and reproducible 96-well plate-based method for the formation of fungal biofilms and its application to antifungal susceptibility testing. Nat Protoc 3:1494-1500.

14. Borman AM, Szekely A, Johnson EM. 2016. Comparative pathogenicity of United Kingdom isolates of the emerging pathogen Candida auris and other key pathogenic Candida species. mSphere 1:e00189-16.

15. Sherry L, Ramage G, Kean R, Borman A, Johnson EM, Richardson MD, RautemaaRichardson R. 2017. Biofilm-forming capability of highly virulent, multidrug-resistant Candida auris. Emerg Infect Dis 23:328-331. 
16. Romera D, Aguilera-Correa JJ, Gadea I, Viñuela-Sandoval L, García-Rodríguez J, Esteban J. 2019. Candida auris: A comparison between planktonic and biofilm susceptibility to antifungal drugs. J Med Microbiol 68:1353-1358.

17. Kean R, Delaney C, Sherry L, Borman A, Johnson EM, Richardson MD, RautemaaRichardson R, Williams C, Ramage G. 2018. Transcriptome assembly and profiling of Candida auris reveals novel insights into biofilm-mediated resistance. mSphere 3:e00334-18.

18. Dominguez EG, Zarnowski R, Choy HL, Zhao M, Sanchez H, Nett JE, Andes DR. 2019. Conserved role for biofilm matrix polysaccharides in Candida auris drug resistance. mSphere 4:e00680-18.

19. Pathirana RU, Friedman J, Norris HL, Salvatori O, McCall AD, Kay J, Edgerton M. 2018. Fluconazole-resistant Candida auris is susceptible to salivary histatin 5 killing and to intrinsic host defenses. Antimicrob Agents Chemother 62:e01872-17.

20. R Core Team. 2021. R: A language and environment for statistical computing. $\mathrm{R}$ Foundation for Statistical Computing, Vienna, Austria.

21. Wickham H, Averick M, Bryan J, Chang W, McGowan LD, François R, Grolemund G, Hayes A, Henry L, Hester J, Kuhn M, Pedersen TL, Miller E, Bache SM, Müller K, Ooms J, Robinson D, Seidel DP, Spinu V, Takahashi K, Vaughan D, Wilke C, Woo K, Yutani H. 2019. Welcome to the tidyverse. J Open Source Softw 4:1686. 
Table 1. Strains used in this study

\begin{tabular}{|c|c|c|c|c|c|c|c|}
\hline \multirow{2}{*}{ Strain no. } & \multirow{2}{*}{$\begin{array}{c}\text { AR Bank } \\
\#\end{array}$} & \multirow{2}{*}{$\begin{array}{l}\text { Geographic } \\
\text { clade }^{\mathrm{a}}\end{array}$} & \multirow{2}{*}{ Aggregation ${ }^{\mathrm{b}}$} & \multicolumn{4}{|c|}{$\mathrm{MIC}(\mathrm{mg} / \mathrm{L})^{\mathrm{a}}$} \\
\hline & & & & $\begin{array}{c}\text { Amphotericin B } \\
(\geq 2 \mathrm{mg} / \mathrm{L})\end{array}$ & $\begin{array}{c}\text { Caspofungin } \\
(\geq 2 \mathrm{mg} / \mathrm{L})\end{array}$ & \begin{tabular}{|c|} 
Fluconazole \\
$(\geq 32 \mathrm{mg} / \mathrm{L})$
\end{tabular} & Flucytosine \\
\hline CAU-01 & 0381 & II (East Asia) & No & 0.38 & 0.125 & 4 & 2 \\
\hline CAU-02 & 0382 & I (South Asia) & Yes & 0.38 & 0.5 & 16 & 0.125 \\
\hline CAU-03 & 0383 & III (South Africa) & Yes & 0.38 & 0.25 & 128 & 0.5 \\
\hline CAU-04 & 0384 & III (South Africa) & Yes & 0.5 & 16 & 128 & 0.5 \\
\hline CAU-05 & 0385 & $\begin{array}{l}\text { IV (South } \\
\text { America) }\end{array}$ & Yes & 0.5 & 0.5 & $>256$ & 0.5 \\
\hline CAU-06 & 0386 & $\begin{array}{l}\text { IV (South } \\
\text { America) }\end{array}$ & Yes & 0.5 & 0.5 & $>256$ & 0.5 \\
\hline CAU-07 & 0387 & I (South Asia) & No & 0.75 & 0.25 & 8 & 8 \\
\hline CAU-08 & 0388 & I (South Asia) & No & 1.5 & 1 & $>256$ & 0.125 \\
\hline CAU-09 & 0389 & I (South Asia) & No & 4 & 0.5 & 256 & 128 \\
\hline
\end{tabular}

${ }^{a}$ From the CDC \& FDA Antibiotic Resistance (AR) Isolate Bank Candida auris (CAU) panel

\section{3 (https://wwwn.cdc.gov/ARIsolateBank/Panel/PanelDetail?ID=2). MICs obtained by broth}

234 microdilution. CDC's tentative breakpoints for each drug are provided (CDC. Candida auris:

235 information for laboratorians and health professionals. Atlanta, Georgia: US Department of

236 Health and Human Services, CDC; 2019. https://www.cdc.gov/fungal/candida-auris/health-

237 professionals.html), and resistance highlighted. 
A.

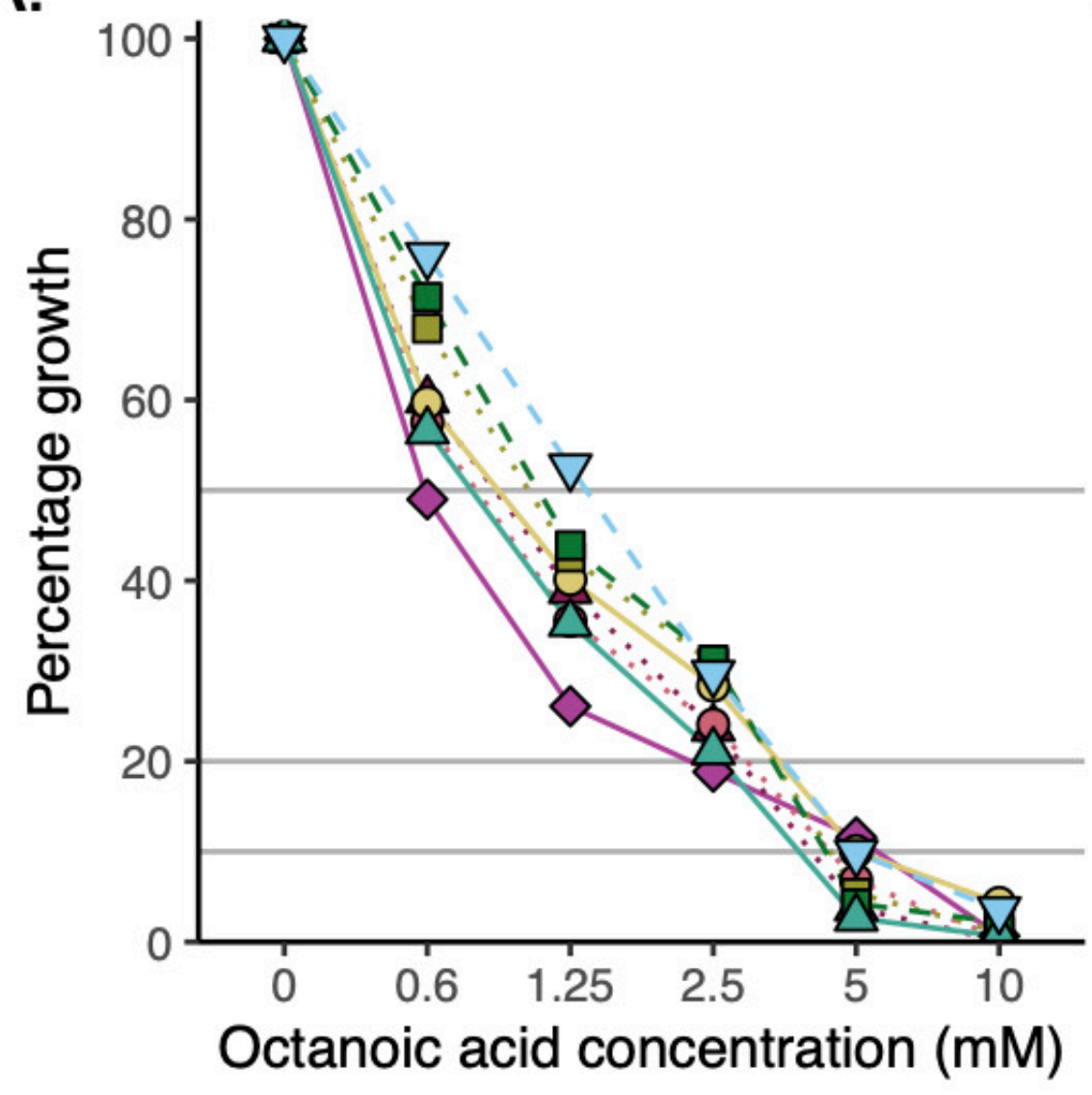

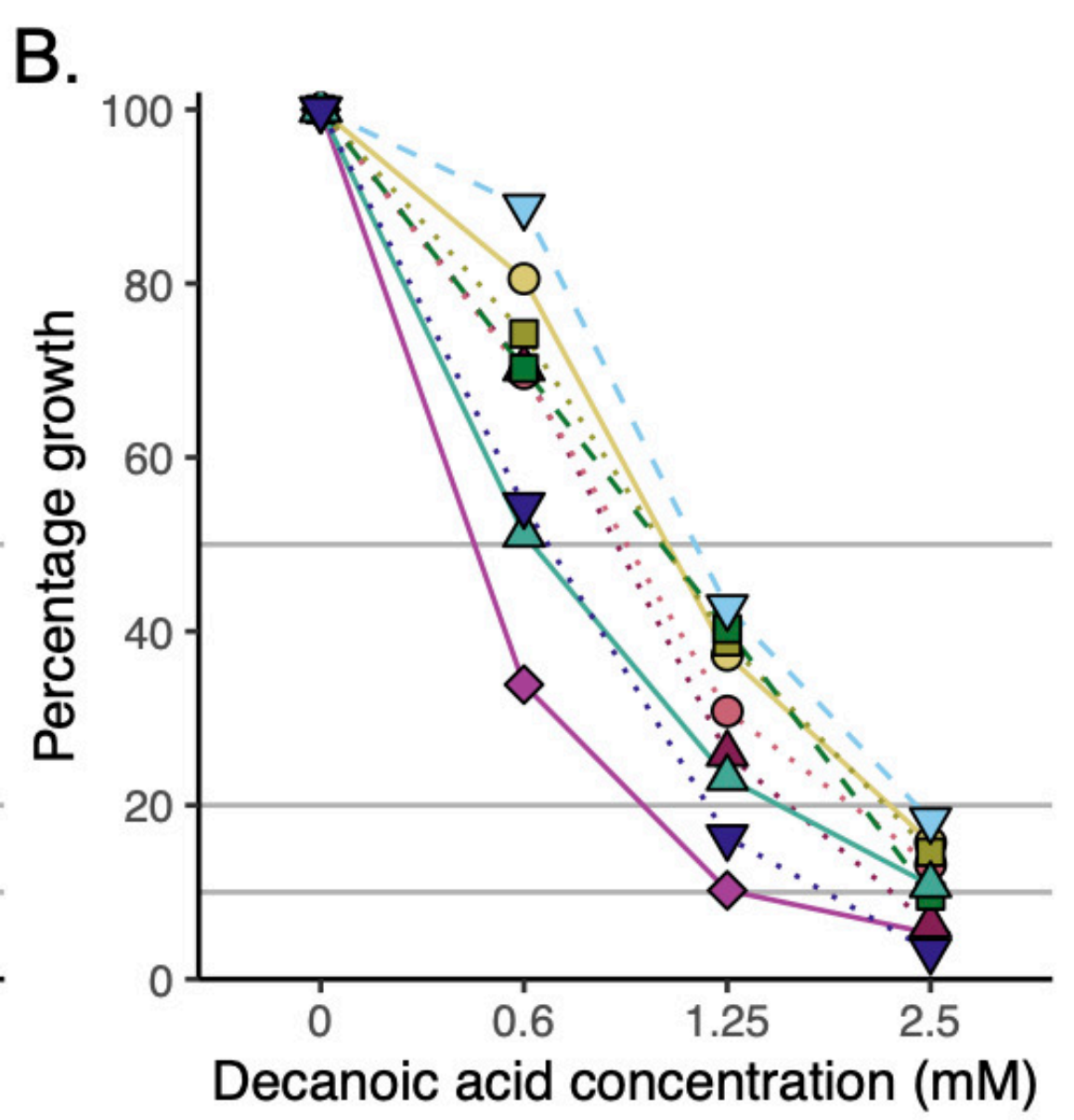

Strain

$\checkmark$ CAU-01

. $\triangle$. CAU-02

. O . CAU-03

O- CAU-04

ㅁ. CAU-05

- $\square-\quad$ CAU-06

$\triangle \quad$ CAU-07

${ }^{-} \nabla^{-} \quad$ CAU-08

' $\nabla \cdot$ CAU-09 


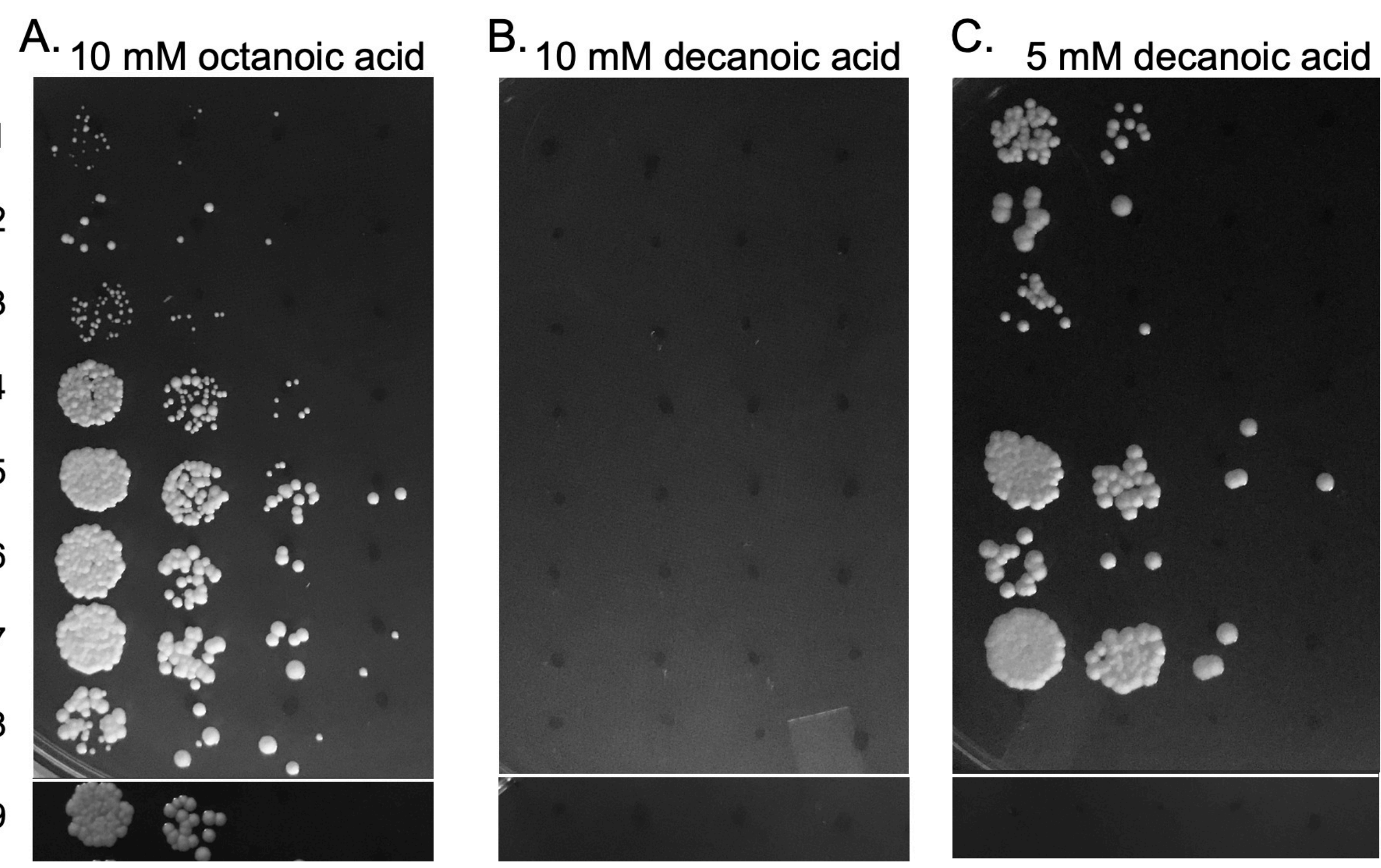


A.

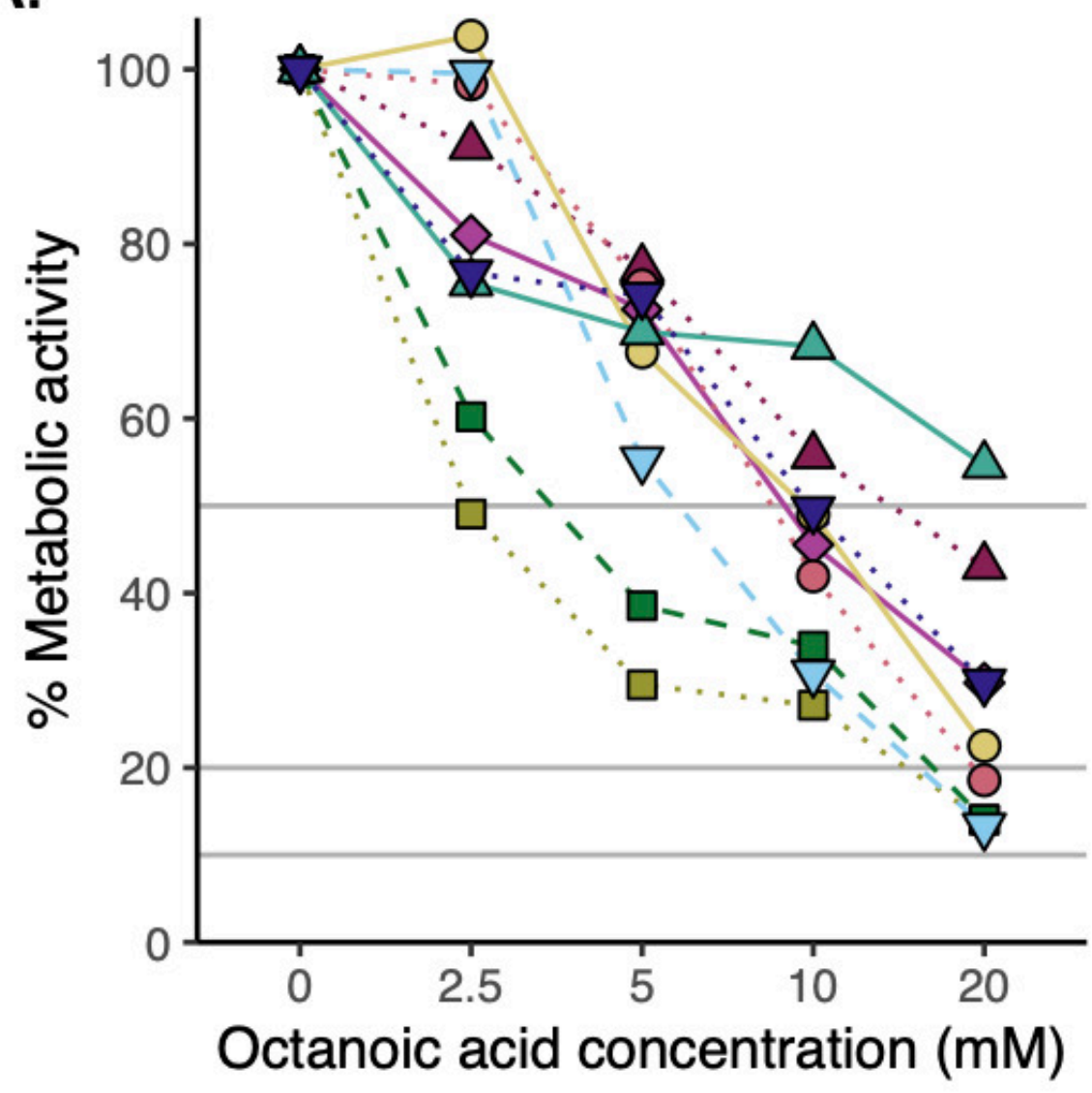

B.

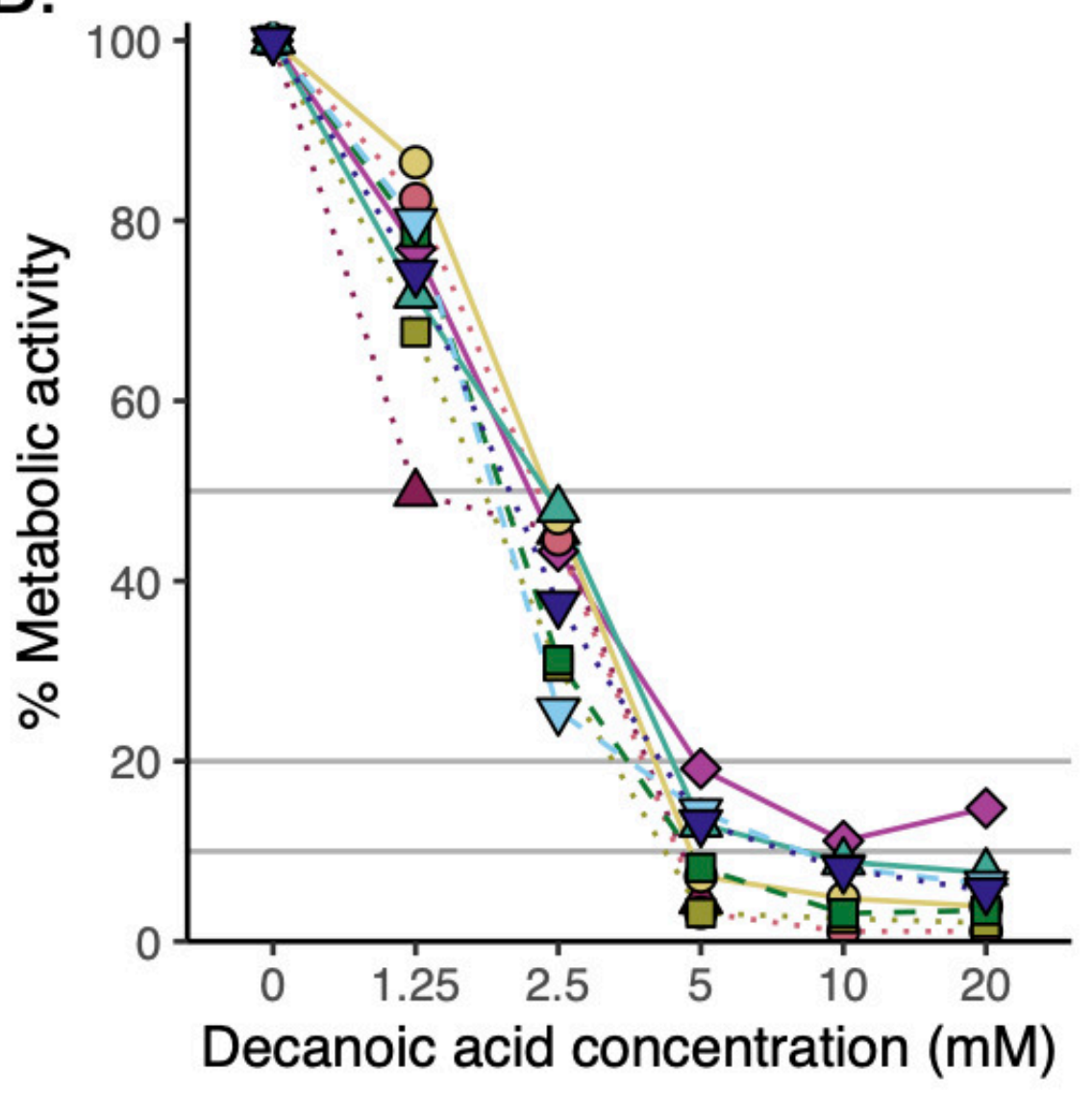

Strain

$\checkmark$ CAU-01

. $\triangle$. CAU-02

O. CAU-03

O- CAU-04

- ‥ CAU-05

- $\square$ - CAU-06

$\triangle \quad$ CAU-07

$-\nabla^{-} \quad$ CAU-08

$\cdot \nabla \cdot$ CAU-09 